\title{
Discussion of 006405JOR by A. Zaccone et al.
}

Citation: Journal of Rheology 58, 1245 (2014); doi: 10.1122/1.4892816

View online: https://doi.org/10.1122/1.4892816

View Table of Contents: http://sor.scitation.org/toc/jor/58/5

Published by the The Society of Rheology

\section{Articles you may be interested in}

Linking self-assembly, rheology, and gel transition in attractive colloids Journal of Rheology 58, 1219 (2014); 10.1122/1.4878838

Stress localization, stiffening, and yielding in a model colloidal gel Journal of Rheology 58, 1089 (2014); 10.1122/1.4882021

A micro-mechanical study of coarsening and rheology of colloidal gels: Cage building, cage hopping, and Smoluchowski's ratchet

Journal of Rheology 58, 1121 (2014); 10.1122/1.4892115

Discussion of 019405JOR by R. Zia et al.

Journal of Rheology 58, 1158 (2014); 10.1122/1.4892821

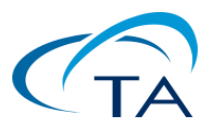

The WORLD'S most

VERSATILE platform for RHEOLOGICAL MEASUREMENTS

The Discovery Hybrid Rheometer
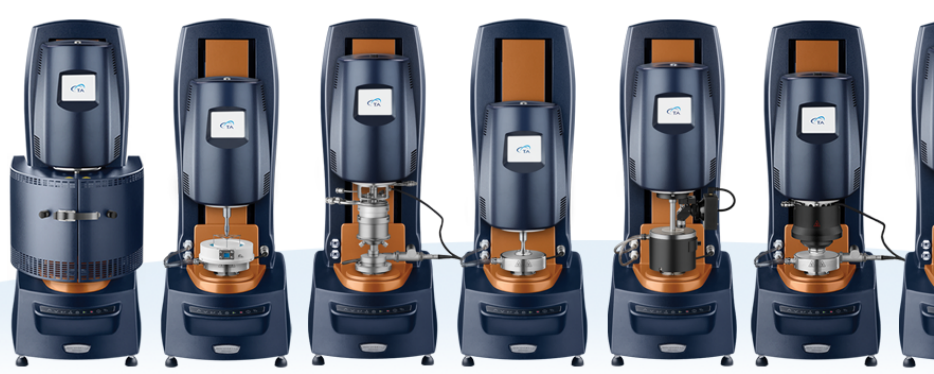


\section{Discussion of 006405JOR by A. Zaccone et al.}

Question: Marco Laurati: The calculation of the relaxation time spectrum that is used to calculate the rheological properties of the gels derives, according to the theory, from the form of the cluster mass (size) distribution. Did you try to compare the predictions of the theory for this distribution with experimental data, for example, obtained using confocal microscopy?

Author Response: Alessio Zaccone: The comparison between theoretical cluster mass distributions and the experimental ones from confocal microscopy is very welcome because it will provide a stringent benchmark to test theoretical predictions based on our approach. We are not aware of such experimental data in the literature. We are very much looking forward to collaborating with experimental groups interested in providing these data. 